\title{
Nonlinear Dynamic Behaviour Analysis of a Clutch System with Uncertainties Using Polynomial Chaos and the Constrained Harmonic Balance Method
}

\author{
M.-H. Trinh, ${ }^{1}$ S. Berger $\mathbb{D}^{2}{ }^{2}$ and E. Aubry ${ }^{3}$ \\ ${ }^{1}$ Department of Automotive Engineering, Hanoi University of Science and Technology, No. 1 Dai Co Viet Street, Hanoi, Vietnam \\ ${ }^{2}$ INSA Centre Val de Loire, Université d'Orléans, Université de Tours, Laboratoire de Mécanique Gabriel Lamé, \\ 3 rue de La Chocolaterie, CS 23410, 41034, Blois Cedex, France \\ ${ }^{3}$ Laboratoire MIPS, Université de Haute-Alsace, 12 rue des Frères Lumière, 68093 Mulhouse Cedex, France
}

Correspondence should be addressed to S. Berger; sebastien.berger@insa-cvl.fr

Received 6 September 2019; Accepted 8 May 2020; Published 26 May 2020

Academic Editor: F. Viadero

Copyright (C) 2020 M.-H. Trinh et al. This is an open access article distributed under the Creative Commons Attribution License, which permits unrestricted use, distribution, and reproduction in any medium, provided the original work is properly cited.

\begin{abstract}
The study of the nonlinear dynamic behaviour of friction systems in general and of clutch systems in particular remains an open problem. Noise and vibrations induced by friction in the sliding phase of a clutch are very sensitive to design parameters. The latter have significant dispersions. In the study of the system stability, the problem is not only to know if the parameter values lead to the appearance of unstable equilibrium points; the real challenge lies in estimating the vibration levels when such unstable equilibrium points occur. This estimation is analyzed using the limit cycles. This article aims to study the ability of robust approaches based on developments in nonintrusive generalized polynomial chaos and a constrained harmonic balance method to estimate the vibration levels through the limit cycles of a clutch system in the presence of uncertainty. The purpose is to provide a low-cost, high precision approach, compared to the classic Monte Carlo method.
\end{abstract}

\section{Introduction}

In the sliding phase of clutch systems in vehicles, self-oscillations may be caused by frictional forces and thereby generate noise. These phenomena can be classified into two main categories depending on whether they are related to tribological aspects or to the geometric and structural characteristics of the systems [1]. For high-frequency oscillations, such as squeal noise (up to several $\mathrm{kHz}$ ), the mode coupling instabilities inherent in the structure of the system are more likely to be responsible for this phenomenon [2]. Therefore, the system stability is determined from the eigenvalues whose real parts are used to analyze the system stability, while the imaginary parts give the frequency of the corresponding modes.

Numerous studies have shown that the dynamic behaviour of dry friction systems in general and clutch systems in particular is very sensitive to design parameters. These studies focused on the analysis of the system stability from the eigenvalues. For example, the effects of friction and damping on the phenomenon of mode coupling in a finite element squeal model of a brake were presented in studies by G. Fritz $[3,4]$. For clutch systems, B. Hervé also studied the effects of these parameters on stability, focusing on the destabilizing paradox [5]. In addition, design parameters such as the friction coefficient and damping admit significant dispersions which may be due inter alia to the manufacturing process. It is therefore necessary to take account of the dispersion of uncertain parameters to ensure the robustness of the analysis of the dynamic behaviour of friction systems. However, the Monte Carlo (MC) method which is conventionally used to achieve this requires prohibitive calculation time, especially for systems with many degrees of freedom (DOF).

The study of the dynamic behaviour of friction systems consists of two steps which are, respectively, the analysis of the 
system stability and the determination of vibration levels when the system is unstable. The present study will focus on the second step. The vibration levels can be estimated through the limit cycles which are generally determined from a temporal integration of differential equations of the motion system. However, this is a difficult and costly process for industrial systems with many DOF (such as the finite element model). To overcome the insufficiencies of the conventional numerical integration of nonlinear friction systems, alternative solutions have been proposed in the literature, such as the central variety method, the complex nonlinear modes method, or the harmonic balance method [4]. Similarly, to take uncertain parameters into account, other methods based on polynomial chaos have been proposed [6]. However, few studies have tackled the problem of the prohibitive costs due to both the consideration of the uncertainties (Monte Carlo method) and temporal integration in friction systems. For example, Nechak et al. $[7,8]$ combined the central variety method with polynomial chaos to take uncertainties into account in initial conditions terms. Similarly, Sarrouy [6] associated the constrained harmonic balance method with polynomial chaos in a braking system. However, these studies have three major limitations. First, the models used only allow two DOF. Second, the number of uncertain parameters is very low (1 or 2$)$. Third, in Sarrouy's study, polynomial chaos is used in an intrusive approach. This approach can easily be applied to simple systems but becomes very slow for systems with many DOF. However, the literature contains no works in which the dynamic behaviour of a clutch system has been studied through the limit cycles, with the consideration of parametric uncertainties.

Therefore, the main objective of this article is to explore the possibility of an approach combining Nonintrusive Generalized Polynomial Chaos (ngPC) and the constrained harmonic balance method (CHBM) to take into account of the uncertainties in the estimation of limit cycles of a clutch system with an increasing number of uncertain parameters. The results are compared with the classic Monte Carlo approach for validation. The aim is to propose an effective method for determining the dispersion of limit cycles at a low cost and with high accuracy in order to overcome the difficulties of the time integration method and the classic MC method.

The novelty of this paper lies in the demonstration of the ability of the methods (CHBM + ngPC) to properly estimate the dispersion of limit cycles of a clutch system with an increasing number of uncertain parameters. Another main interest of this paper is the results obtained with the study of uncertain clutch system.

This paper is organized as follows: Section 2 presents a squeal model of a clutch system, Section 3 is dedicated to the theoretical foundations of the methods applied in the estimation of the system's limit cycles, and the results and discussions of the limit cycles analysis are given in Section 4, followed by a conclusion in Section 5 .

\section{Squeal Model of the Clutch System}

2.1. System Description. In friction systems with unstable modes, the limit cycles evaluation is rather delicate. $\mathrm{Nu}$ merical integration often leads to an increase in the vibration levels which does not exist in practice [9]. In the works dedicated to the study of an analytical model of an aircraft braking system with $15 \mathrm{DOF}$, how the presence of nonlinearities in the model helps to obtain limit cycles during the vibration levels analysis with unstable modes was shown [10]. In the model, the nonlinearities occur in the normal contact forces in terms of stiffness with a polynomial law of an order 3 displacement. It is important to note that this model was validated in an experimental approach.

In another study [11] dedicated to a clutch system with 2 DOF, the author used a nonlinear model of the normal contact forces between the flywheel and the friction disc to obtain the limit cycles. In this model defined from experimental studies, the normal forces also possess a nonlinear polynomial of an order 3 displacement.

Otherwise, Wickramarachi and Singh [2] proposed a model of a clutch system with 6 DOF to study the instabilities due to mode couplings induced by friction. This model based on experiments helped to study the system's stability state from an analysis with eigenvalues. However, it is not possible to estimate the vibration levels as limit cycles cannot be obtained with this model. Therefore, this paper proposes to extend the Wickramarachi model by introducing a nonlinear part in the normal contact forces in terms of stiffness with an order 3 displacement $\left(k_{A}^{\mathrm{NL}}, k_{B}^{\mathrm{NL}}, k_{C}^{\mathrm{NL}}, k_{D}^{\mathrm{NL}}\right)$ and a damping part to take account of the damping differences in the system $\left(c_{A}, c_{B}, c_{C}, c_{D}\right)$ (Figure 1).

The model used in this paper is a mass/spring model with 6 DOF. The contact between the flywheel and the friction surface of the clutch disk is determined at points $A^{\prime}, B^{\prime}, C^{\prime}$, and $D^{\prime}$ by a progressive spring $k p$ which is divided into 4 stiffnesses $k_{A}, k_{B}, k_{C}$, and $k_{D}$. Dampings $c_{A}, c_{B}, c_{C}$, and $c_{D}$ are placed, respectively, in the same positions as the springs $k_{A}$, $k_{B}, k_{C}$, and $k_{D}$. Points $A, B, C$, and $D$ are the projections of contacts on the average surface of the pressure plate. The pressure plate is deformable and modelled by the bending stiffness and damping $\left(k_{f}, c_{f}\right)$. Points $E, F, G$, and $H$ are fixed points of the flywheel.

In a state of static equilibrium, progressive stiffnesses $k_{A}$, $k_{B}, k_{C}$, and $k_{D}$ are assumed to be static at a constant value $k_{p} / 4$. Due to the vibration of the pressure plate around the axis $\mathrm{O} x$, points $A$ and $B$ vibrate with low amplitudes, and the stiffnesses $k_{A}$ and $k_{B}$ vary according to a hyperbolic curve around a constant value $k_{p} / 4$. The stiffnesses $k_{A}$ and $k_{B}$ are then, respectively, multiplied and divided by a ratio $\gamma 1$. Similarly, the stiffnesses $k_{C}$ and $k_{D}$ are, respectively, multiplied and divided by a ratio $\gamma 1$ (see (6) and (7)) [2].

To model the nonlinear forces in the clutch system, the cubic nonlinear stiffnesses $k_{A}^{\mathrm{NL}}, k_{B}^{\mathrm{NL}}, k_{C}^{\mathrm{NL}}, k_{D}^{\mathrm{NL}}$ are introduced in the same positions as those of the linear stiffnesses $k_{A}, k_{B}$, $k_{C}$, and $k_{D}$ (see (8)).

The DOF of the pressure plate are the rotations $\theta x$ and $\theta y$ around the fixed axes $x$ and $y$ and the translation movements $Z A, Z B, Z C$, and $Z D$ of points $A, B, C$, and $D$ along the fixed axis $z$.

The described equation of the dynamic behaviour of the clutch system is 

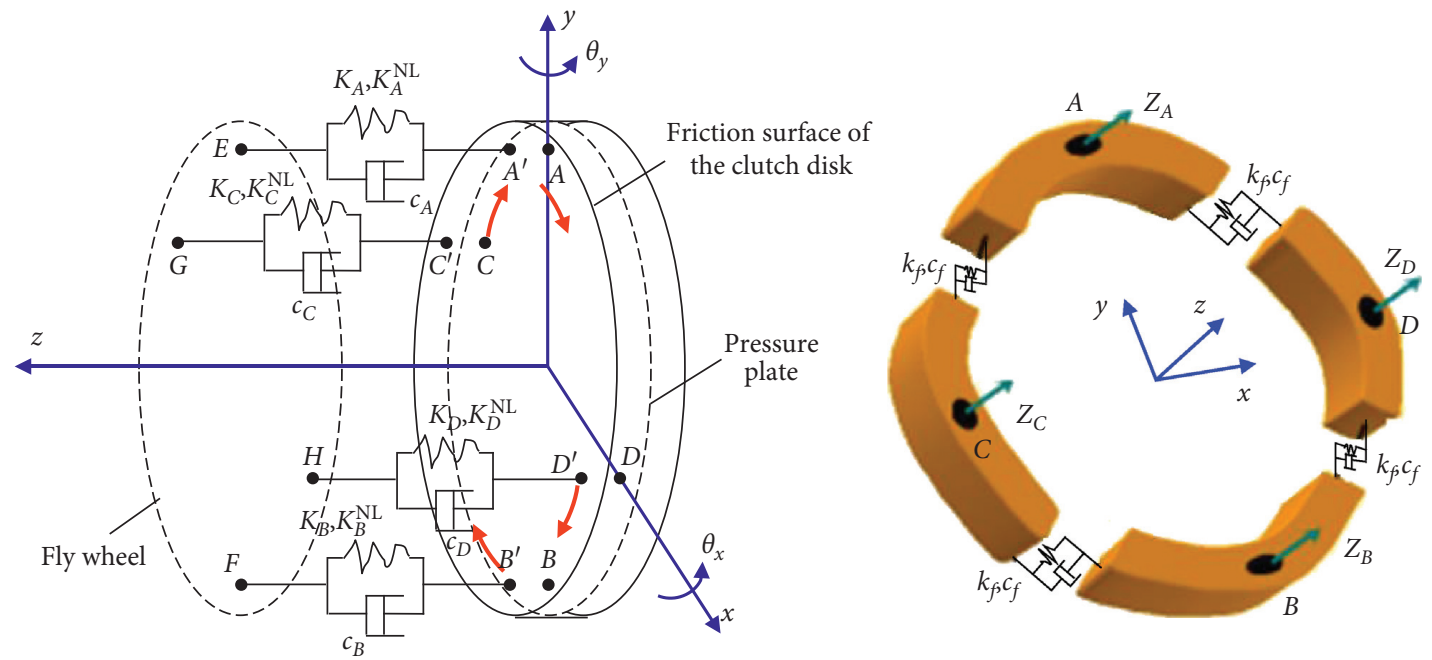

FIgURE 1: Squeal model of the clutch system.

$$
\mathbf{M} \frac{\mathrm{d}^{2} \mathbf{u}}{\mathrm{d} t^{2}}+\mathbf{C} \frac{\mathrm{d} \mathbf{u}}{\mathrm{d} t}+\mathrm{Ku}+\mathbf{F}_{\mathrm{NL}}=0
$$

with

$$
\begin{aligned}
& \mathbf{u}=\left[\begin{array}{llllll}
\theta_{x} & \theta_{y} & Z_{A} & Z_{B} & Z_{C} & Z_{D}
\end{array}\right]^{\mathrm{T}}, \\
& \mathbf{M}=\left[\begin{array}{cccccc}
I_{x} & 0 & 0 & 0 & 0 & 0 \\
0 & I_{y} & 0 & 0 & 0 & 0 \\
0 & 0 & \frac{M_{p}}{4} & 0 & 0 & 0 \\
0 & 0 & 0 & \frac{M_{p}}{4} & 0 & 0 \\
0 & 0 & 0 & 0 & \frac{M_{p}}{4} & 0 \\
0 & 0 & 0 & 0 & 0 & \frac{M_{p}}{4}
\end{array}\right] \text {, } \\
& \mathbf{K}=\left[\begin{array}{cccccc}
r^{2}\left(k_{A}+k_{B}+4 k_{f}\right) & -\mu l r\left(k_{C}+k_{D}\right) & r\left(k_{A}+2 k_{f}\right) & -r\left(k_{B}+2 k_{f}\right) & \mu l k_{C} & -\mu l k_{D} \\
\mu l r\left(k_{A}+k_{B}\right) & r^{2}\left(k_{C}+k_{D}+4 k_{f}\right) & -\mu l k_{A} & \mu l k_{B} & r\left(k_{C}+2 k_{f}\right) & -r\left(k_{D}+2 k_{f}\right) \\
r\left(k_{A}+2 k_{f}\right) & 0 & k_{A}+2 k_{f} & 0 & -k_{f} & -k_{f} \\
-r\left(k_{B}+2 k_{f}\right) & 0 & 0 & k_{B}+2 k_{f} & -k_{f} & -k_{f} \\
0 & r\left(k_{C}+2 k_{f}\right) & -k_{f} & -k_{f} & k_{C}+2 k_{f} & 0 \\
0 & -r\left(k_{D}+2 k_{f}\right) & -k_{f} & -k_{f} & 0 & k_{D}+2 k_{f}
\end{array}\right] \text {, } \\
& \mathbf{C}=\left[\begin{array}{cccccc}
r^{2}\left(c_{A}+c_{B}+4 c_{f}\right) & -\mu l r\left(c_{C}+c_{D}\right) & r\left(c_{A}+2 c_{f}\right) & -r\left(c_{B}+2 c_{f}\right) & \mu l c_{C} & -\mu l c_{D} \\
\mu l r\left(c_{A}+c_{B}\right) & r^{2}\left(c_{C}+c_{D}+4 c_{f}\right) & -\mu l c_{A} & \mu l c_{B} & r\left(c_{C}+2 c_{f}\right) & -r\left(c_{D}+2 c_{f}\right) \\
r\left(c_{A}+2 c_{f}\right) & 0 & c_{A}+2 c_{f} & 0 & -c_{f} & -c_{f} \\
-r\left(c_{B}+2 c_{f}\right) & 0 & 0 & c_{B}+2 c_{f} & -c_{f} & -c_{f} \\
0 & r\left(c_{C}+2 c_{f}\right) & -c_{f} & -c_{f} & c_{C}+2 c_{f} & 0 \\
0 & -r\left(c_{D}+2 c_{f}\right) & -c_{f} & -c_{f} & 0 & c_{D}+2 c_{f}
\end{array}\right],
\end{aligned}
$$




$$
\begin{aligned}
& k_{A}=\frac{\gamma_{1} \cdot k_{p}}{4} ; \\
& k_{B}=\frac{k_{p}}{\gamma_{1} \cdot 4}, \\
& k_{C}=\frac{\gamma_{2} \cdot k_{p}}{4} ; \\
& k_{D}=\frac{k_{p}}{\gamma_{2} \cdot 4},
\end{aligned}
$$

where $r=\left(r_{1}+r_{2}\right) / 2$ with $r_{1}$ and $r_{2}$ which are the minimal and maximal sliding radii; $\mu$ is the friction coefficient; and $l$ is the half thickness of the pressure plate.
Nonlinear force FNL is given in the following equation:

$$
\mathbf{F}_{\mathrm{NL}}=\left[\begin{array}{c}
r k_{A}^{\mathrm{NL}}\left(Z_{A}+r \theta_{x}\right)^{3}-r k_{B}^{\mathrm{NL}}\left(Z_{B}-r \theta_{x}\right)^{3}+\mu l k_{C}^{\mathrm{NL}}\left(Z_{C}+r \theta_{y}\right)^{3}-\mu l k_{D}^{\mathrm{NL}}\left(Z_{D}-r \theta_{y}\right)^{3} \\
-\mu l k_{A}^{\mathrm{NL}}\left(Z_{A}+r \theta_{x}\right)^{3}+\mu l k_{B}^{\mathrm{NL}}\left(Z_{B}-r \theta_{x}\right)^{3}+r k_{C}^{\mathrm{NL}}\left(Z_{C}+r \theta_{y}\right)^{3}-r k_{D}^{\mathrm{NL}}\left(Z_{D}-r \theta_{y}\right)^{3} \\
k_{A}^{\mathrm{NL}}\left(Z_{A}+r \theta_{x}\right)^{3} \\
k_{B}^{\mathrm{NL}}\left(Z_{B}-r \theta_{x}\right)^{3} \\
k_{C}^{\mathrm{NL}}\left(Z_{C}+r \theta_{y}\right)^{3} \\
k_{D}^{\mathrm{NL}}\left(Z_{D}-r \theta_{y}\right)^{3}
\end{array}\right] .
$$

2.2. Configurations of Uncertain Parameters. Different configurations of uncertain parameters will be addressed in Section 4 , each serving a different goal. The dispersions of the parameters $V_{i}$ are assumed according to uniform laws. To follow the formalism of polynomial chaos, each of these parameters $V_{i}$ is expressed in the functions of independent random variables $\xi_{i}$ which follow a uniform distribution in the interval $[-1,1]$ such that

$$
V_{i}=V_{m, i}+\Delta V_{m, i} \xi_{i},
$$

where $V_{m, i}$ and $\Delta V_{m, i} \xi_{i}$ denote the nominal value and the variation of uncertain parameters $V_{i}$.

For the studies of the limit cycles of the system, the 8 uncertain parameters $\mu, k_{p}, k_{f}, \gamma 1, \gamma 2, r 1, r 2$, and $l$ are supposed uniform in the intervals $\left[V_{m, i}-V_{m, i} * \Delta V_{m, i} V_{m, i}+\right.$ $\left.V_{m, i} * \Delta V_{m, i}\right]$ to ensure an unstable static equilibrium for the entire interval. All of the nonlinear stiffnesses and dampings are assumed to be constant (Table 1).

\section{Constrained Harmonic Balance Method and Nonintrusive Generalized Polynomial Chaos Applied to the Clutch System}

3.1. Constrained Harmonic Balance Method (CHBM). The self-excited nonlinear system studied in this paper tends to lead to limit cycles when static equilibrium is unstable. The system, being nonlinear, converges toward limit cycles whose amplitude and frequency are unknown. To determine their properties while avoiding a long direct integration process, a well-known method is the harmonic balance method which consists of a Galerkin approach using basic trigonometric functions [12]. This method relies on the decomposition of the solution $\mathbf{u}(t)$ in a Fourier series which is truncated to a given order $p 1$ :

$$
\mathbf{u}(t)=\mathbf{A}_{0}+\sum_{k=1}^{p_{1}}\left(\mathbf{A}_{\mathbf{k}} \cos (k \omega t)+\mathbf{B}_{k} \sin (k \omega t)\right),
$$

where $\omega=2 \pi / \tau$ defines the fundamental frequency (unknown period) of the limit cycles of nonlinear system. $\mathbf{A}_{k}$ and $\mathbf{B}_{k}$ are real vectors of the same size as $\mathbf{u}(t)$. This evolution is also imposed for $\mathrm{d} \mathbf{u}(t) / \mathrm{d} t$ and $\mathrm{d}^{2} \mathbf{u}(t) / \mathrm{d} t^{2}$.

Once these truncated Fourier series are reinjected into dynamic (1), the time variable is eliminated by the projection system of equations on the functions $(1, \cos (k \omega t)$, and $\sin (k \omega t))$ using the following scalar product:

$$
\langle f, g\rangle=\frac{2}{T} \int_{t=0}^{T} f(t) g(t) d t .
$$

This generates a square equation system of size $n$ $\left(2 p_{1}+1\right)$ with $n$ the number of DOF of the system.

However, when the angular frequency $\omega$ is unknown, the system is not square and it is generally necessary to add an equation or to discard an unknown. The first solution is exploited in a study [4] by adding a constraint on the real parts of the eigenvalues of the system but this procedure requires a great amount of calculations and does not correspond to evolution when uncertainties are introduced. Therefore, a study in [13] aims to explore the second solution 
TABLE 1: Nominal values of the parameters of the clutch system with 6 DOF.

\begin{tabular}{lcc}
\hline Parameter name & Unit & Nominal value \\
\hline Friction coefficient $(\mu)$ & & $0.25 ; 0.35 ; 0.5$ \\
Progressive stiffness $\left(k_{p}\right)$ & $\mathrm{MN} \cdot \mathrm{m}^{-1}$ & 16 \\
Bending stiffness $\left(k_{f}\right)$ & $\mathrm{MN} \cdot \mathrm{m}^{-1}$ & 7 \\
Coefficient $(\gamma 1)$ & & 0.9 \\
Coefficient $(\gamma 2)$ & & 0.8 \\
Minimum radius of flywheel $(r 1)$ & $\mathrm{m}$ & 0.075 \\
Maximum radius of flywheel $(r 2)$ & $\mathrm{m}$ & 0.120 \\
Half thickness of flywheel $(l)$ & $\mathrm{m}$ & 0.0125 \\
Nonlinear stiffness $\left(k_{A}^{\mathrm{NL}}\right)$ & $\mathrm{MN} \cdot \mathrm{m}^{-3}$ & $4 * 105$ \\
Nonlinear stiffness $\left(k_{B}^{\mathrm{NL}}\right)$ & $\mathrm{MN} \cdot \mathrm{m}^{-3}$ & $12 * 105$ \\
Nonlinear stiffness $\left(k_{C}^{\mathrm{NL}}\right)$ & $\mathrm{MN} \cdot \mathrm{m}^{-3}$ & 4 \\
Nonlinear stiffness $\left(k_{D}^{\mathrm{NL}}\right)$ & $\mathrm{MN}^{-3} \mathrm{~m}^{-3}$ & 4 \\
Progressive damping $\left(c_{p}\right)$ & $\mathrm{Nm}{ }^{-1} \cdot \mathrm{s}^{-1}$ & 4 \\
Bending damping $\left(c_{f}\right)$ & $\mathrm{Nm}{ }^{-1} \cdot \mathrm{s}^{-1}$ & 0.1 \\
\hline
\end{tabular}

by eliminating an unknown and fixing it at a given value. The method is then called constrained harmonic balance method $(\mathrm{CHB})$. This is an interesting improvement of the usual procedure of this method which provides a means to determine the limit cycles of nonlinear systems.
For the clutch system with $6 \mathrm{DOF}, \mathbf{u}(\mathrm{t})$ is developed with a single harmonic of an unknown frequency $(p 1=1)$ and without a constant term (for the central solution approximation only), and an unknown is eliminated by assigning a given value $a_{1}^{*}$ to the first component of vector a:

$$
\begin{aligned}
\mathbf{u}(t) & =\mathbf{a} \cos (\omega t)+\mathbf{b} \sin (\omega t), \\
\mathbf{a} & =\left[\begin{array}{llllll}
a_{1}^{*} & a_{2} & a_{3} & a_{4} & a_{5} & a_{6}
\end{array}\right]^{\mathrm{T}}, \\
\mathbf{b} & =\left[\begin{array}{llllll}
b_{1} & b_{2} & b_{3} & b_{4} & b_{5} & b_{6}
\end{array}\right]^{\mathrm{T}} .
\end{aligned}
$$

Injecting the harmonic expression (see (12)) and its derivatives in the dynamic (1) and projecting the resulting set of equations onto $\cos (\omega t)$ and $\sin (\omega t)$ functions using scalar product (see (11)), one finally gets a set of 12 algebraic equations:

$$
\left\{\begin{array}{c}
\left(\mathbf{K}-\omega^{2} \mathbf{M}\right) \mathbf{a}+\omega \mathbf{C} \mathbf{b}+\mathbf{F}_{\mathrm{NL}, \mathrm{cos}}=\mathbf{0} \\
\left(\mathbf{K}-\omega^{2} \mathbf{M}\right) \mathbf{b}-\omega \mathbf{C a}+\mathbf{F}_{\mathrm{NL}, \sin }=\mathbf{0}
\end{array}\right.
$$

with

$$
F_{\mathrm{NL}, \mathrm{cos}}=\left[\begin{array}{c}
r k_{A}^{\mathrm{NL}}\left[\left(a_{3}+r a_{1}^{*}\right)^{3}+\left(a_{3}+r a_{1}^{*}\right)\left(b_{3}+r b_{1}\right)^{2}\right]-r k_{B}^{\mathrm{NL}}\left[\left(a_{4}-r a_{1}^{*}\right)^{3}+\left(a_{4}-r a_{1}^{*}\right)\left(b-r b_{1}\right)^{2}\right] \\
+\mu l k_{C}^{\mathrm{NL}}\left[\left(a_{5}+r a_{2}\right)^{3}+\left(a_{5}+r a_{2}\right)\left(b_{5}+r b_{2}\right)^{2}\right]-\mu l k_{D}^{\mathrm{NL}}\left[\left(a_{6}-r a_{2}\right)^{3}+\left(a_{6}-r a_{2}\right)\left(b_{6}-r b_{2}\right)^{2}\right] \\
-\mu l k_{A}^{\mathrm{NL}}\left[\left(a_{3}+r a_{1}^{*}\right)^{3}+\left(a_{3}+r a_{1}^{*}\right)\left(b_{3}+r b_{1}\right)^{2}\right]+\mu l k_{B}^{\mathrm{NL}}\left[\left(a_{4}-r a_{1}^{*}\right)^{3}+\left(a_{4}-r a_{1}^{*}\right)\left(b_{4}-r b_{1}\right)^{2}\right] \\
+r k_{C}^{\mathrm{NL}}\left[\left(a_{5}+r a_{2}\right)^{3}+\left(a_{5}+r a_{2}\right)\left(b_{5}+r b_{2}\right)^{2}\right]-r k_{D}^{\mathrm{NL}}\left[\left(a_{6}-r a_{2}\right)^{3}+\left(a_{6}-r a_{2}\right)\left(b_{6}-r b_{2}\right)^{2}\right] \\
k_{A}^{\mathrm{NL}}\left[\left(a_{3}+r a_{1}^{*}\right)^{3}+\left(a_{3}+r a_{1}^{*}\right)\left(b_{3}+r b_{1}\right)^{2}\right] \\
k_{B}^{\mathrm{NL}}\left[\left(a_{4}-r a_{1}^{*}\right)^{3}+\left(a_{4}-r a_{1}^{*}\right)\left(b_{4}-r b_{1}\right)^{2}\right] \\
k_{C}^{\mathrm{NL}}\left[\left(a_{5}+r a_{2}\right)^{3}+\left(a_{5}+r a_{2}\right)\left(b_{5}+r b_{2}\right)^{2}\right] \\
k_{D}^{\mathrm{NL}}\left[\left(a_{6}-r a_{2}\right)^{3}+\left(a_{6}-r a_{2}\right)\left(b_{6}-r b_{2}\right)^{2}\right]
\end{array}\right],
$$

The algebraic equations (13) which have 12 unknown variables $\omega, b_{1}, a_{2}, b_{2}, a_{3}, b_{3}, a_{4}, b_{4}, a_{5}, b_{5}, a_{6}$, and $b_{6}$ are solved with the function "fsolve" in the Matlab software.

3.2. Nonintrusive Generalized Polynomial Chaos Method $(n g P C)$. The dispersions of the uncertain parameters generate the dispersions in the displacements $u_{i}(t, \xi)$, the velocities $\dot{u}_{i}(t, \xi)$, and so the dispersions of the unknown variables $\omega(\xi)$ and $b_{i}(\xi)$ of the CHB method. These quantities of interest will be estimated from the Legendre polynomial chaos which is best adapted to the treatment of uniform uncertainties [7]. 


$$
y(\xi)=\sum_{j=0}^{N_{p}} \bar{y}_{j} \phi_{j}(\xi)
$$

where $\bar{y}_{j}$ are the stochastic modes, $\phi_{j}$ are the Legendre polynomials, and $N_{p}$ is the number of terms according to the order $p$ of the polynomial chaos and the number of uncertain parameters $r$, such that

$$
N_{p}+1=\frac{(p+r) !}{p ! r !} .
$$

The representation with the generalized polynomial chaos requires the determination of $N_{p}+1$ stochastic modes (see (16)). Two implementation patterns may be used: an intrusive approach and a nonintrusive approach. In the first approach, a Galerkin projection technique is used to generate a set of deterministic coupled equations from the stochastic model, which keeps the same properties as the original random functions. The calculation of stochastic coefficients then passes through the adaptation of a calculation algorithm corresponding to the nature of the resulting equation system. The interest of the intrusive approach is that only one calculation is required to determine the stochastic modes $[6,13]$. However, this becomes rather expensive and/or impossible, particularly when systems are highly nonlinear in many DOF with several important uncertain parameters. In this case, the second nonintrusive approach becomes an attractive alternative, as it allows the calculation of the stochastic modes without any alteration or modification of the original uncertain model. The difficulty is only related to the direct simulation of the original system in order to obtain the $Q$ points which are called the collocation points needed to build the stochastic modes. Two methods can be used to determine these points Q: the nonintrusive spectral projection method (NISP) and the regression method $[6-8,12,14-16]$. The authors have shown that the best points are those constructed from the roots of the Legendre polynomial of order $p+1$. If we choose the same order $p$ for $r$ random variables, the number of points is $(p+1)^{r}$.

In terms of calculation amount, the regression approach offers an interesting alternative in particular with respect to the NIPS method $[6,12,15,16]$. The authors have shown that the $Q$ collocation points to construct stochastic modes using the regression method can be selected from the $(p+1)^{r}$ points determined from roots of the Legendre polynomial of order $p+1$, with $Q$ equal to $k N_{p}(k=2,3)$. The present study proposes to choose the $Q$ collocation points according to the following criteria:

$$
Q=\min \left((p+1)^{r}, 2 N_{p}\right) .
$$

Thus, the stochastic modes are estimated using the regression method by minimizing, in the least square sense, the difference $\varepsilon$ between the solution $y$ of the stochastic model and its approximation in the base of polynomial chaos:

$$
\varepsilon=\sum_{q=1}^{Q}\left[y\left(\xi^{(q)}\right)-\sum_{l=0}^{N_{p}} \bar{y}_{l} \cdot \varphi_{l}\left(\xi^{(q)}\right)\right]^{2},
$$

where $\xi(q)$ are the $Q$ collocations points determined from the roots of the Legendre polynomial $\varphi_{l}$ of order $p+1$ according the criteria (see (18)).

\section{Results and Discussion}

The coalescence phenomena which generate the system instability occur between two wobbling modes, numbered 1 and 2, which correspond to the angular displacements $\theta x$ and $\theta y$. Therefore, the study of the limit cycles of the system will focus on these two modes only. Note that, in the system, the limit cycles followed by $\theta x$ and $\theta y$ are similar, so only the associated results with $\theta x$ will be shown.

In this section, some studies are presented in which the limit cycles are determined, on a sample of $N=100$ parameter sets. The objective is to determine the dispersion of the limit cycles due to the dispersion of the uncertain parameters. This number of sample $N$ was chosen to be sufficiently high so as to provide representative results of the system behaviour, but not too high either, to ensure reasonable calculation time. In these studies, the limit cycles are calculated using different methods:

(i) Method 1: the displacements $u_{i}(t, \xi)$, velocities $\dot{u}_{i}(t, \xi)$, and limit cycles are calculated by the direct solution of the initial complete system (see (1)) through a Monte Carlo type study (results 1).

(ii) Method 2: the displacements $u_{i}(t, \xi)$, velocities $\dot{u}_{i}(t, \xi)$, and limit cycles are modelled using the developments in generalized polynomial chaos (ngPC) (see (19)):

$$
\begin{aligned}
& u_{i}(t, \xi)=\sum_{j=0}^{N_{p}} \overline{u_{i j}(t)} \varphi_{j}(\xi) \\
& \dot{u}_{i}(t, \xi)=\sum_{j=0}^{N_{p}} \overline{\dot{u}_{i j}(t)} \varphi_{j}(\xi) .
\end{aligned}
$$

To do so, the stochastic modes $\overline{u_{i j}(t)}$ and $\overline{\dot{u}_{i j}(t)}$ of these ngPC are determined from displacements and velocities obtained with the regression method from direct simulation of the initial complete system (see (1)). The limit cycles are then calculated from the ngPC (see (19)) (results 2). The objective is to determine the ability of applied ngPC on the displacements and velocities to estimate the limit cycles of the system.

(iii) Method 3: the $\left(\omega(\xi), a_{i}(\xi), b_{i}(\xi)\right)$ coefficients of the CHBM are calculated directly from equation (see (13)). The limit cycles are then calculated from the equations (see (12)). Results 3 are obtained with Monte Carlo simulations on coefficients of the CHBM. The objective is to determine the ability of the CHBM to estimate the limit cycles of the system. 
(iv) Method 4: the $\left(\omega(\xi), a_{i}(\xi), b_{i}(\xi)\right)$ coefficients of the CHBM are modelled using the developments in generalized polynomial chaos (see (20)):

$$
\begin{aligned}
& a_{i}(\xi)=\sum_{j=0}^{N_{p}} \bar{a}_{i j} \varphi_{j}(\xi) ; \\
& b_{i}(\xi)=\sum_{j=0}^{N_{p}} \bar{b}_{i j} \varphi_{j}(\xi) ; \\
& \omega(\xi)=\sum_{j=0}^{N_{p}} \bar{\omega}_{j} \varphi_{j}(\xi) .
\end{aligned}
$$

To do so, $\left(\overline{a_{i j}}, \overline{b_{i j}}, \omega_{j}\right)$ stochastic modes of these ngPC are determined with the regression method from the values of the $\left(\omega(\xi), a_{i}(\xi), b_{i}(\xi)\right)$ coefficients of the CHBM obtained by solving the system of (13). The limit cycles are then calculated from (12) and the ngPC coefficients 21 (results 4). The objective of this study is to determine the ability of the combination of CHBM and ngPC to estimate the limit cycles of the system.

The results obtained with the last three methods are compared for validation to those obtained with the first one used as a reference. The three methods are then compared with each other in the terms of accuracy and computation time to determine the most effective one.

The comparisons between the different results are made on the evolution of the limit cycles and the relative errors of the maximum and minimum amplitudes of the displacements $u_{i}(t, \xi)$ and velocities $\dot{u}_{i}(t, \xi)$.

In the studies, the friction coefficient $\mu \mathrm{m}$ will be selected with 3 values at increasingly higher distances of the Hopf bifurcation point: $\mu m=\mu 0(1+\varepsilon)$ with $\varepsilon=1.27(\mu m=0.25)$, $\varepsilon=2.18(\mu m=0.35)$, and $\varepsilon=4.54(\mu m=0.50)$. From the Hopf bifurcation point, the real part of a mode is positive and the static equilibrium becomes unstable $[14,16]$. Note that the Hopf bifurcation point is $\mu 0=0.11$.

4.1. Study of the Limit Cycles of the System with One Uncertain Parameter. This section presents a study of the limit cycles of the system with one uncertain parameter $(r=1)$. The friction coefficient is selected in $N=100$ points in the interval $\left[\mu_{m}-\mu_{m} \Delta \mu, \mu_{m}+\mu_{m} \Delta \mu\right]$ with $\mu m=0.25$ and $\Delta \mu=0.05$ (5\%); the other parameters are fixed at their nominal value. The limit cycles are estimated using the four methods described above. The objective of this study is to evaluate the ability of these methods to estimate the dispersion of the limit cycles of the system, in terms of accuracy and computational costs.

First, we compare these methods in terms of calculation costs for $N$ sets of parameters (Table 2). Each calculation time written in Table 2 has been evaluated with one set of parameters and allows reader to have an estimation of the order of magnitude for the total calculation cost. The calculation time of stochastic modes is, respectively, $41 \mathrm{~s}$ for ngPC which is applied to displacements and velocities and is $7 e-4$ seconds for ngPC which is applied to the coefficients of the CHBM. The calculation time for the determination of the coefficients of the CHBM is one to two seconds for one set of parameters. One limit cycle determined with a ngPC development is obtained in $3.96 \mathrm{~s}$ with previously determined stochastic ngPC modes. The time of calculation of one cycle limit obtained with the CHBM is negligible (less than $2.10^{-3} \mathrm{~s}$ ) when the coefficients are previously determined. Except for the calculation time for the determination of the coefficients of the CHBM which depends on the number of DOF of the initial system, these calculation times do not directly depend on the number of DOF of the system studied. Thus, they are of the same order of magnitude with the clutch system with 6 DOF or with a model with a high number of DOF. However, to obtain one limit cycle by performing a direct simulation takes about $17.71 \mathrm{~s}$ with the clutch system with $6 \mathrm{DOF}$, but it can take several hours for an industrial model (finite element model) with several hundreds of DOF. So, if these methods are to be applied to an industrial model, the comparison of the different methods in terms of calculation costs also needs the comparison of the required number $\mathrm{N}$ of direct simulations (limit cycles) with the initial model (column 1 of Table 2). If this number is the same for both methods, the times intrinsically required for the methods (the calculations of stochastic modes for ngPC; the determination of coefficients for the CHBM) can then be compared.

In results 2, the displacements and velocities are directly modelled with the ngPC (see (19)). The stochastic modes are determined using the regression method. Here, the order $p$ of ngPC should be equal to 30 to be accurate (the related errors of frequencies and amplitudes of the displacements and velocities are below 10\%). In the case of a single uncertain parameter, only 31 simulations of the complete system are required to build the stochastic modes. Similarly, $3.96 \mathrm{~s}$ only is required to determine a limit cycle for one set of parameters. ngPC (particularly with the regression method) is efficient in terms of calculation costs.

In results 3 , the displacements and velocities are determined using the CHBM. It takes $1.43 \mathrm{~s}$ to determine the coefficients of the CHBM and a negligible time (less than $2.10^{-3} \mathrm{~s}$ ) to determine one limit cycle. The CHBM is therefore more efficient in terms of calculation costs than ngPC.

In results 4 , the displacements and velocities are determined using the CHBM (see (12)) but with coefficients modelled using ngPC (see (20)). To obtain the necessary accuracy with ngPC, an order $p=2$ is sufficient. So, for one uncertain parameter, only three resolutions of the system (see (13)) are sufficient to determine the stochastic modes of the coefficients of the CHBM and it just takes $1.98 \mathrm{~s}$ to calculate them. It must be noted that once the stochastic modes are calculated, it is not necessary to solve the system of equations (see (13)). The CHBM (see (12)) provides the limit cycles in a negligible time (less than $2.10^{-3} \mathrm{~s}$ ). Thus, the CHBM combined with the ngPC is much more efficient in terms of calculation costs than ngPC alone, the CHBM alone, or the direct MC method. As no simulation of the initial system is necessary, this performance is even more pronounced when the number of samples is high. In the present study, 100 sets of 


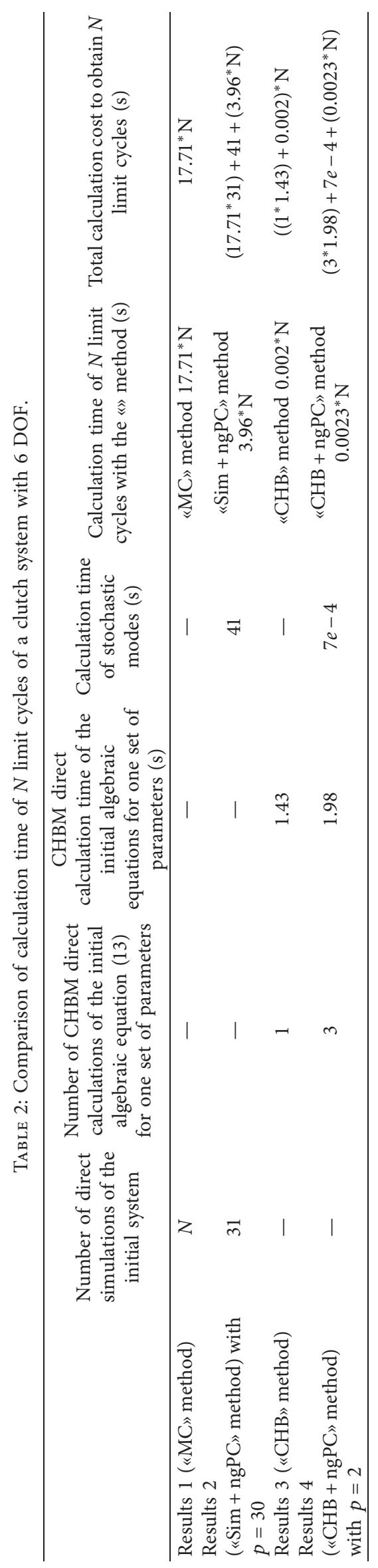


parameters were calculated, but it may be necessary to perform 1,000 or 10,000 calculations.

Figure 2 shows the relative errors, in percentage, of maximum and minimum frequencies $(\omega)$ and of maximum and minimum amplitudes of the displacements $\left(\theta_{1}\right)$ and velocities $\left(\mathrm{d} \theta_{1}\right)$ between the direct calculation $(\mathrm{MC})$ and the $\mathrm{CHBM}+$ ngPC according to the order $p$ of the chaos ( $p=1$ to $10)$. The relative errors of the frequencies are very low $(<0.3 \%)$. The relative errors of the amplitudes are less than $10.5 \%$. In addition, the relative errors remain constant when $p$ increases. The error is in this case due to the limited accuracy of the CHBM (see (13)) in reproducing the solution of (1). Therefore, order $p=2$ is selected.

Figure 3 shows the dispersion of 100 calculated limit cycles due to the dispersion of the uncertain parameters, with the 4 methods: direct simulation (MC-a-green), ngPC for displacement and velocities with order $p=30$ (Sim + ngPC-b-green), constrained harmonic balance (CHBM-a-blue), and constrained harmonic balance with ngPC for the coefficients with order $p=2$ (CHBM + ngPC-b-blue). The calculated limit cycles, respectively, MC-a-green and Sim + CHBM-b-green, are virtually coincident. Similarly, the limit cycles determined, respectively, with CHBM-a-blue and $\mathrm{CHBM}+$ ngPC-b-blue are also very close. These results show that the use of ngPC, directly either for the displacements and velocities or for the coefficients of the CHBM, does not harm the precision of the limit cycles. However, a slight difference is observed between $\mathrm{Sim}+$ ngPC-b-green and CHBM+ngPC-b-blue curves and between MC-a-green and CHBM-a-blue curves. This shows that the use of CHBM whether with or without ngPC generates a relatively small error (Table 3 ).

The results obtained with the 4 methods are compared in Table 3 . They show the maximum and minimum amplitudes of the displacements and velocities and their relative errors in comparison with MC. The relative errors between the results calculated with MC and Sim + ngPC are very close $(<1 \%)$. The relative errors results, respectively, obtained with the CHBM and with the combination of CHBM and ngPC, in comparison with $\mathrm{MC}$, are similar and below $10 \%$.

Finally, among the different methods studied, the method based on the combination of the CHBM and ngPC is the most efficient one in terms of calculation costs and great accuracy.

4.2. Study of the Limit Cycles of the System with Several Uncertain Parameters. In the studies below, several uncertain parameters (from 1 to 8 ) are taken into account in the estimation of the limit cycles of the system. The objective is to evaluate the ability of the method based on the combination of the CHBM and ngPC to estimate the limit cycles with an increasing number of uncertain parameters. The results obtained are compared with those obtained with the classic MC method applied to the initial system. In addition, the influence of the nominal values and the width of the interval dispersions of the uncertain parameters are investigated.
4.2.1. Study of the Limit Cycles with Different Nominal Values of the Friction Coefficient. The objective of this section is to investigate, respectively, the influence of the nominal value $\mu$ of the friction coefficient and the dimension of the width of the uncertainty parameters intervals other than that of the friction coefficient $\Delta \mu$ on the effectiveness of the method based on the CHBM and ngPC to estimate the limit cycles of the clutch system.

In this study, the friction coefficient is again chosen in $N=100$ random points of the interval $\left[\mu_{m}-\mu_{m} \Delta \mu, \mu_{m}+\mu_{m} \Delta \mu\right]$ with three different nominal values of the friction coefficient $\mu m=0.25 ; 0.35$; and 0.5 and with the variation $\Delta \mu=0.05$ (5\%). These values correspond to points which are increasingly farther from the Hopf bifurcation point $\mu 0$ (here $\mu 0=0.11$ ). The other parameters are considered as uncertain randoms ( $N=100$ points) in the intervals $\left[V_{m, i}-V_{m, i} \Delta V_{m, i}, V_{m, i}+V_{m, i} \Delta V_{m, i}\right], V_{m, i}$ is the nominal value of the $i^{\text {th }}$ uncertain parameter, and $\Delta V_{m, i}$ is successively equal to $0.01(1 \%)$ and $0.05(5 \%)$.

The number of uncertain parameters is successively equal to $1,2,5$, and 8 . The order of the polynomial chaos used is equal to 2 . The results are given for the three nominal values of the friction coefficient (Table 4).

In terms of calculation costs, it should be noted that the number of resolutions for the system of equations (see (13)) ranges from 3 to 90 .

The comparison values are the maximum and minimum amplitudes of displacement and velocity which are used to determine the dispersion of the limit cycles. The dispersion of the limit cycles naturally increases with the number of uncertain parameters. For each value of the coefficient, the relative errors for the maximum and minimum amplitudes of displacement and velocity vary and slightly increase with the number of uncertain parameters.

For $\Delta V_{m, i}=0.01$, when $\mu m$ increases (farther from the Hopf bifurcation point), the relative errors will decrease (from 7 to $10 \%$ for $\mu m=0.25$, from 3 to $6 \%$ for $\mu m=0.35$, and from 1 to $4 \%$ for $\mu m=0.50$ ). Therefore, the calculation precision of the limit cycles is increasingly high.

With $\mu m=0.25$, the fact of increasing $V_{m, i}$ to 0.05 instead of 0.01 implies small errors (7 to 11\%) for a number of uncertain parameters $r<5$ but leads to important errors beyond (14\% to $37 \%$ ). The method is then no longer effective. With $\mu m=0.35$, for any uncertainty interval, the relative errors lie in the range of 4 to $7 \%$. Similarly, with $\mu m=0.5$ and for any uncertainty interval, the relative errors lie in the range of 1 to $4 \%$. Thus, the combination of the constrained harmonic balance method and ngPC is more effective for the estimation of the limit cycles of the system as the area is far from the Hopf bifurcation point. This is because the vibration levels increase in the same way. These results are confirmed in Figure 4 which represents the limit cycles of the system determined with $(\mathrm{CHB}+\mathrm{gPC})$ for three values of the friction coefficient.

In conclusion, except for the case of $\mu m=0.25$ and the uncertainty intervals admitting a dispersion of $5 \%$, the combination of the CHBM and the ngPC is effective with the intervals of uncertainty parameters other than the friction 


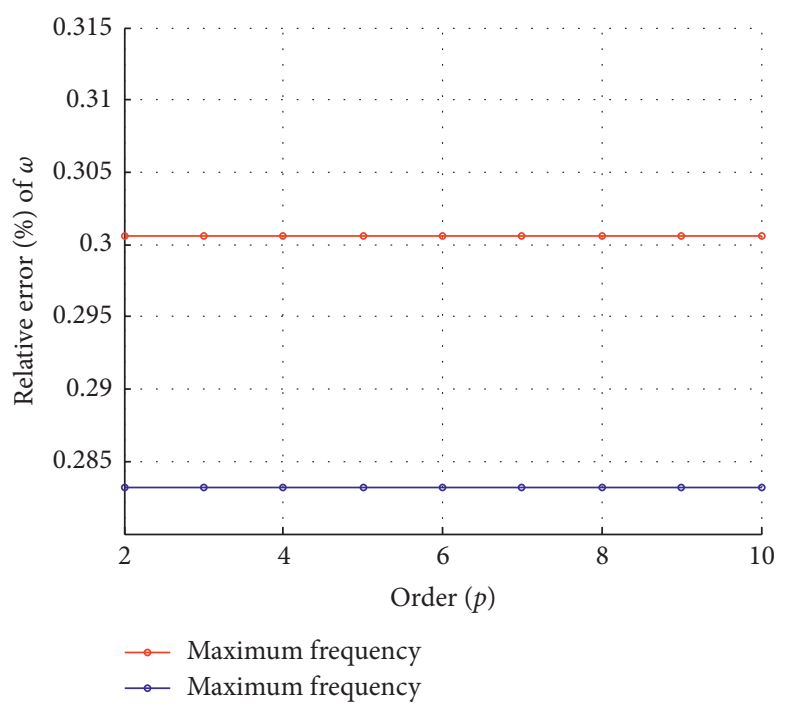

(a)

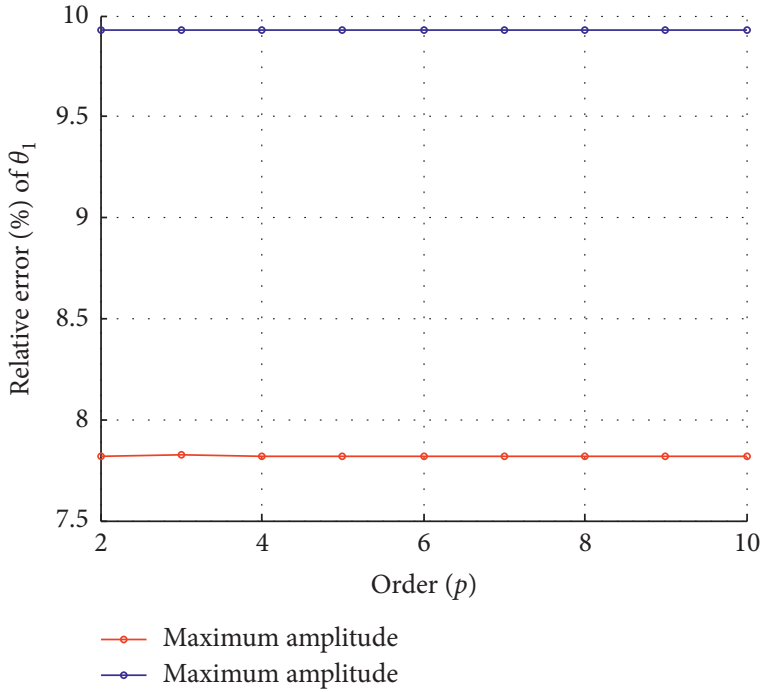

(b)

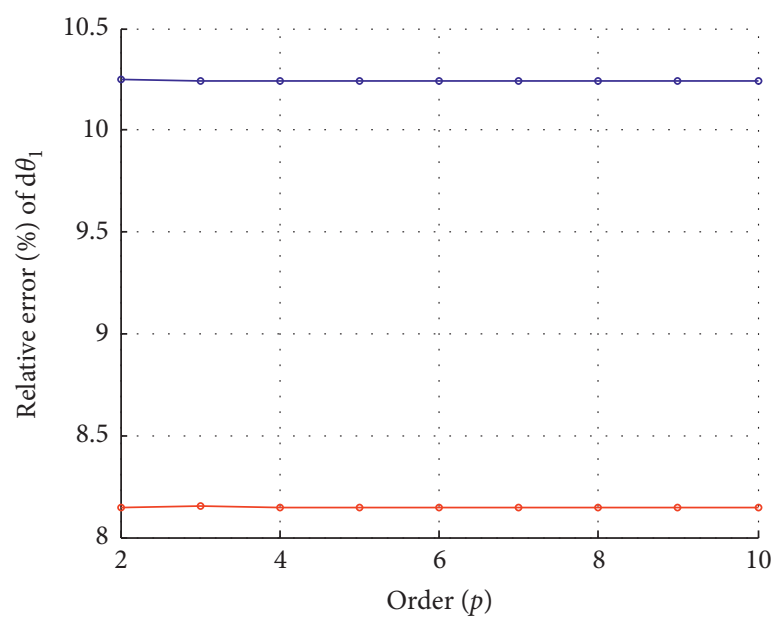

- Maximum amplitude

$\rightarrow$ Maximum amplitude

(c)

FIGURE 2: The relative errors (\%) of the maximum and minimum frequencies $(\omega)$ (a) of the maximum and minimum amplitudes of displacements $\left(\theta_{1}\right)$ and $(\mathrm{b})$ of velocities $\left(\mathrm{d} \theta_{1}\right)$ (c) between the direct calculation $(\mathrm{MC})$ and ngPC + CHBM with order $p$.

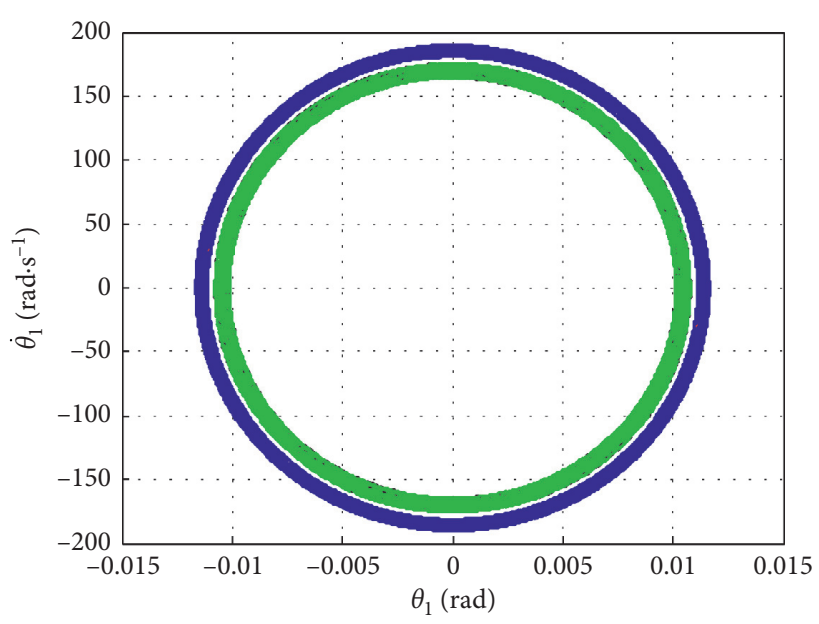

o $\mathrm{CHBM}$

- $\mathrm{MC}$

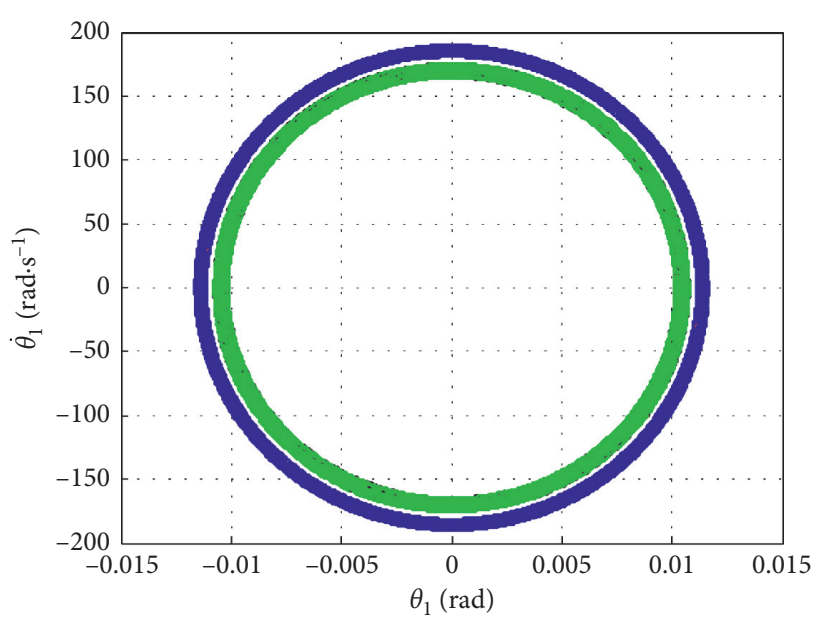

- $\mathrm{CHBM}+$ ngPC

- $\mathrm{Sim}+\mathrm{ngPC}$

FIGURE 3: Dispersion of limit cycles ( $\mu=$ uncertain, $\mu_{\mathrm{m}}=0.25$, and $\Delta \mu=0.05$ ). 
TABLE 3: Comparison of calculated limit cycles of a clutch system with 6 DOF, using different methods.

\begin{tabular}{|c|c|c|c|c|}
\hline Methods & $\mathrm{MC}$ & $\mathrm{Sim}+\mathrm{ngPC}$ & CHBM & $\mathrm{CHBM}+\mathrm{ngPC}$ \\
\hline Maximum amplitude of displacements (rad) & 0.0216 & 0.0216 & 0.0232 & 0.0232 \\
\hline Relative error of the maximum amplitude of displacements (\%) & - & 0.21 & 7.82 & 7.81 \\
\hline Minimum amplitude of displacements (rad) & 0.0204 & 0.0204 & 0.0224 & 0.0224 \\
\hline Relative error of the minimum amplitude of displacements (\%) & - & 0.04 & 9.92 & 9.93 \\
\hline Maximum amplitude of velocities $\left(\mathrm{rads}^{-1}\right)$ & 349.8 & 351.0 & 378.2 & 378.2 \\
\hline Relative error of the maximum amplitude of velocities (\%) & - & 0.38 & 8.14 & 8.14 \\
\hline Minimum amplitude of velocities ( $\mathrm{rad} / \mathrm{s})$ & 330.4 & 330.4 & 364.4 & 364.4 \\
\hline Relative error of the minimum amplitude of velocities (\%) & - & 0.04 & 10.24 & 10.24 \\
\hline
\end{tabular}

TABLE 4: Comparison of the limit cycles of a clutch system with several uncertain parameters between the MC method and the combination of the CHBM and ngPC: three different values of the friction coefficient and with different dispersions interval for uncertain parameters.

\begin{tabular}{|c|c|c|c|c|c|c|c|c|c|}
\hline \multirow{4}{*}{$\mathrm{N}$} & Number of uncertain parameters $r$ & \multicolumn{2}{|c|}{1} & \multicolumn{2}{|c|}{2} & \multirow{2}{*}{\multicolumn{2}{|c|}{$\begin{array}{c}5 \\
\mu, k_{p}, k_{f}, \gamma 1, \\
\gamma 2,\end{array}$}} & \multirow{2}{*}{\multicolumn{2}{|c|}{$\begin{array}{c}8 \\
\mu, k_{p}, k_{f}, \gamma 1, \\
\gamma 2, r_{1}, r_{2}, l\end{array}$}} \\
\hline & & \multicolumn{2}{|c|}{$\mu$} & \multicolumn{2}{|c|}{$\mu, k_{p}$} & & & & \\
\hline & Number of resolutions for the system of equations (see (13)) & & & & & \multicolumn{2}{|c|}{42} & \multicolumn{2}{|c|}{90} \\
\hline & $\Delta V_{m, i}$ & 0.01 & 0.05 & 0.01 & 0.05 & 0.01 & 0.05 & 0.01 & 0.05 \\
\hline \multirow{8}{*}{$\mu=0.25$} & Maximum amplitude of displacements $\left({ }^{*} 10 e-4, \mathrm{rad}\right)$ & 216 & 216 & 216 & 220 & 218 & 254 & 218 & 240 \\
\hline & Relative error of the maximum amplitude of displacements (\%) & 7.81 & 7.81 & 7.72 & 7.13 & 7.64 & 8.16 & 8.07 & 37.7 \\
\hline & Minimum amplitude of displacements $\left({ }^{*} 10 e-, \mathrm{rad}\right)$ & 204 & 204 & 204 & 198 & 200 & 180 & 198 & 168 \\
\hline & Relative error of the minimum amplitude of displacements (\%) & 9.93 & 9.93 & 9.96 & 10.7 & 10.1 & 12.3 & 10.4 & 19.4 \\
\hline & Maximum amplitude of velocities $\left(\mathrm{rad} \cdot \mathrm{s}^{-1}\right)$ & 350 & 350 & 352 & 360 & 354 & 398 & 362 & 410 \\
\hline & Relative error of the maximum amplitude of velocities (\%) & 8.14 & 8.14 & 8.05 & 7.47 & 7.98 & 8.96 & 8.41 & 24.9 \\
\hline & Minimum amplitude of velocities $\left(\mathrm{rad} \cdot \mathrm{s}^{-1}\right)$ & 330 & 330 & 330 & 320 & 324 & 286 & 320 & 274 \\
\hline & Relative error of the minimum amplitude of velocities (\%) & 10.2 & 10.2 & 10.2 & 11.0 & 10.4 & 12.5 & 10.5 & 14.7 \\
\hline \multirow{8}{*}{$\mu=0.35$} & Maximum amplitude of displacements $\left({ }^{*} 10 e-4, \mathrm{rad}\right)$ & 256 & 256 & 256 & 262 & 258 & 276 & 258 & 282 \\
\hline & Relative error of the maximum amplitude of displacements (\%) & 3.73 & 3.73 & 4.02 & 3.44 & 2.89 & 3.18 & 3.87 & 3.35 \\
\hline & Minimum amplitude of displacements $\left({ }^{*} 10 e-4, \mathrm{rad}\right)$ & 244 & 244 & 244 & 238 & 240 & 224 & 236 & 212 \\
\hline & Relative error of the minimum amplitude of displacements (\%) & 4.61 & 4.61 & 4.63 & 4.93 & 4.59 & 5.36 & 5.97 & 6.89 \\
\hline & Maximum amplitude of velocities $\left(\mathrm{rad} \cdot \mathrm{s}^{-1}\right)$ & 416 & 416 & 418 & 426 & 420 & 454 & 428 & 494 \\
\hline & Relative error of the maximum amplitude of velocities (\%) & 4.12 & 4.12 & 4.09 & 3.85 & 3.63 & 3.79 & 4.27 & 4.11 \\
\hline & Minimum amplitude of velocities $\left(\mathrm{rad} \cdot \mathrm{s}^{-1}\right)$ & 396 & 396 & 396 & 384 & 388 & 356 & 386 & 338 \\
\hline & Relative error of the minimum amplitude of velocities (\%) & 4.98 & 4.98 & 4.97 & 5.30 & 5.21 & 5.70 & 5.06 & 5.19 \\
\hline \multirow{8}{*}{$\mu=0.5$} & Maximum amplitude of displacements $\left({ }^{*} 10 e-4, \mathrm{rad}\right)$ & 304 & 304 & 304 & 310 & 306 & 322 & 306 & 330 \\
\hline & Relative error of the maximum amplitude of displacements (\%) & 1.74 & 1.74 & 1.72 & 1.61 & 1.73 & 1.51 & 1.79 & 1.58 \\
\hline & Minimum amplitude of displacements $\left({ }^{*} 10 e-4, \mathrm{rad}\right)$ & 290 & 290 & 290 & 284 & 290 & 272 & 286 & 260 \\
\hline & Relative error of the minimum amplitude of displacements (\%) & 2.16 & 2.16 & 1.90 & 2.30 & 1.07 & 2.41 & 2.64 & 3.07 \\
\hline & Maximum amplitude of velocities $\left(\mathrm{rad} \cdot \mathrm{s}^{-1}\right)$ & 494 & 494 & 496 & 506 & 496 & 528 & 508 & 574 \\
\hline & Relative error of the maximum amplitude of velocities (\%) & 2.26 & 2.26 & 2.24 & 2.14 & 2.26 & 2.18 & 2.24 & 2.41 \\
\hline & Minimum amplitude of velocities $\left(\mathrm{rad} \cdot \mathrm{s}^{-1}\right)$ & 470 & 470 & 470 & 460 & 470 & 434 & 460 & 410 \\
\hline & Relative error of the minimum amplitude of velocities (\%) & 2.64 & 2.64 & 2.21 & 2.76 & 1.21 & 2.85 & 3.65 & 2.63 \\
\hline
\end{tabular}

coefficient, admitting a dispersion of $1 \%$ or $5 \%$, in any of zones of the Hopf bifurcation point compared.

4.2.2. Study of the Limit Cycles with Different Dispersions of the Friction Coefficient. The objective of this study is to investigate the influence of the uncertainty interval dimension of the friction coefficient $\mu$ (the most important parameter in friction systems) on the effectiveness of the method based on the CHBM and ngPC to estimate the limit cycles of the clutch system.

This study is similar to the previous one with $\mu m=0.5$ and $\Delta V_{m, i}=0.01$, considering a variation $\Delta \mu$ equal not only to $0.05(5 \%)$, but also to $0.1(10 \%)$.
Table 5 shows that the relative errors of the minimum and maximum amplitudes of the displacements and velocities obtained with a variation $\Delta \mu$ equal to 0.1 are of the same order of magnitude as those obtained with a variation $\Delta \mu$ equal to 0.05 . When the uncertainty interval increases, the dispersions of the vibration levels naturally increase. Other trends with a variation $\Delta \mu$ equal to 0.1 are the same as those obtained with a variation $\Delta \mu$ equal to 0.05 . The same conclusions can be drawn with a nominal value of the friction coefficient equal to 0.25 or 0.35 (Figure 5).

In conclusion, the use of the combination of the CHBM and ngPC is effective with a dispersion of 0.1 or 0.05 of the friction coefficient, in any of the zones of the Hopf bifurcation point compared. 


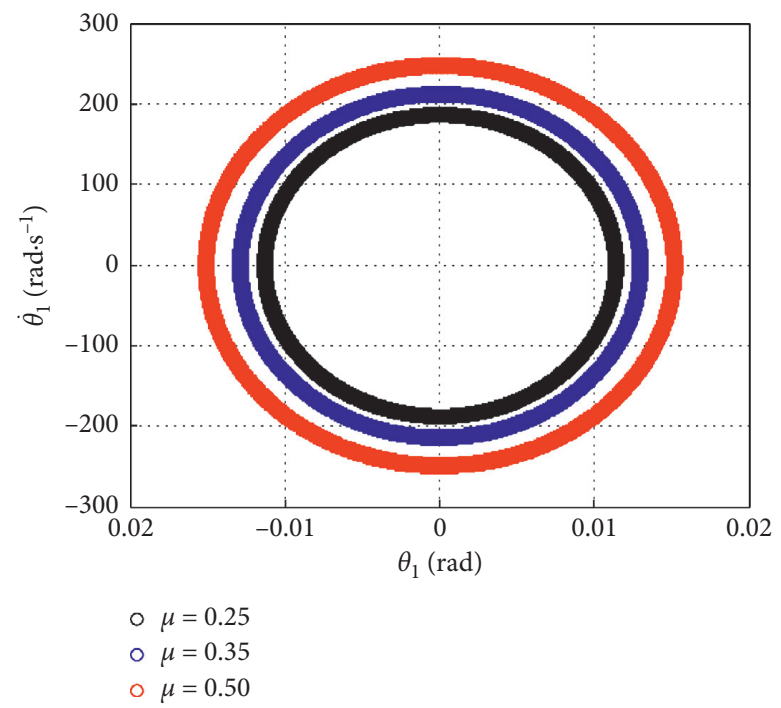

FIgURE 4: Dispersion of the limit cycles determined with $(\mathrm{CHBM}+\mathrm{ngPC})\left(r=8 ; \Delta \mu=0.05 ; \Delta V_{m, i}=0.01 ; \mu m=0.25 ; 0.35 ; 0.50\right)$.

TABLE 5: Comparison of the limit cycles of a clutch system with 6 DOF with different intervals of the friction coefficient $(\mu=0.5$; $\left.\Delta V_{m, i}=0.01\right)$.

\begin{tabular}{|c|c|c|c|c|c|c|c|c|}
\hline \multirow{2}{*}{$\begin{array}{l}\text { Number of uncertain parameters } r \\
\text { Number of resolutions for the system of equations (see (13)) }\end{array}$} & \multicolumn{2}{|c|}{1} & \multicolumn{2}{|c|}{2} & \multicolumn{2}{|c|}{5} & \multicolumn{2}{|c|}{8} \\
\hline & \multicolumn{2}{|c|}{3} & \multicolumn{2}{|c|}{9} & \multicolumn{2}{|c|}{42} & \multicolumn{2}{|c|}{90} \\
\hline Friction coefficient variation $\Delta \mu$ & 0.05 & 0.1 & 0.05 & 0.1 & 0.05 & 0.1 & 0.05 & 0.1 \\
\hline Maximum amplitude of displacements $\left({ }^{*} 10 e-4, \mathrm{rad}\right)$ & 304 & 310 & 304 & 310 & 306 & 310 & 306 & 312 \\
\hline Relative error of the maximum amplitude of displacements (\%) & 1.74 & 1.57 & 1.72 & 1.56 & 1.73 & 2.57 & 1.79 & 1.67 \\
\hline Minimum amplitude of displacements $\left({ }^{*} 10 e-4, \mathrm{rad}\right)$ & 290 & 282 & 290 & 282 & 290 & 280 & 286 & 280 \\
\hline Relative error of the minimum amplitude of displacements (\%) & 2.16 & 2.42 & 1.90 & 2.46 & 2.07 & 2.39 & 2.64 & 2.39 \\
\hline Maximum amplitude of velocities $\left(\mathrm{rads}^{-1}\right)$ & 494 & 504 & 496 & 504 & 496 & 506 & 508 & 516 \\
\hline Relative error of the maximum amplitude of velocities (\%) & 2.26 & 2.10 & 2.24 & 2.10 & 2.26 & 2.11 & 2.24 & 2.21 \\
\hline Minimum amplitude of velocities $\left(\mathrm{rads}^{-1}\right)$ & 470 & 458 & 470 & 456 & 470 & 456 & 460 & 450 \\
\hline Relative error of the minimum amplitude of velocities (\%) & 2.64 & 2.88 & 2.21 & 3.15 & 1.21 & 2.53 & 3.65 & 2.85 \\
\hline
\end{tabular}

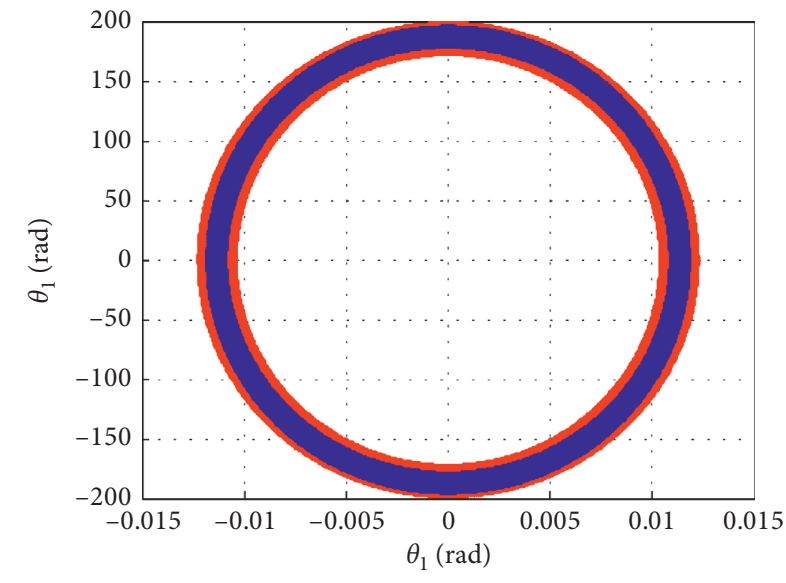

○ $\Delta \mu=0.05$

○ $\Delta \mu=0.10$

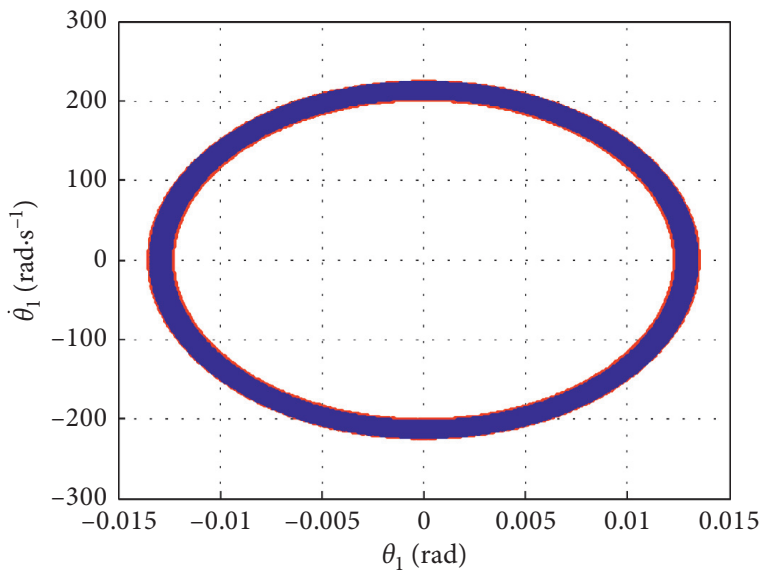

○ $\Delta \mu=0.05$

- $\Delta \mu=0.10$

(a)

Figure 5: Continued. 


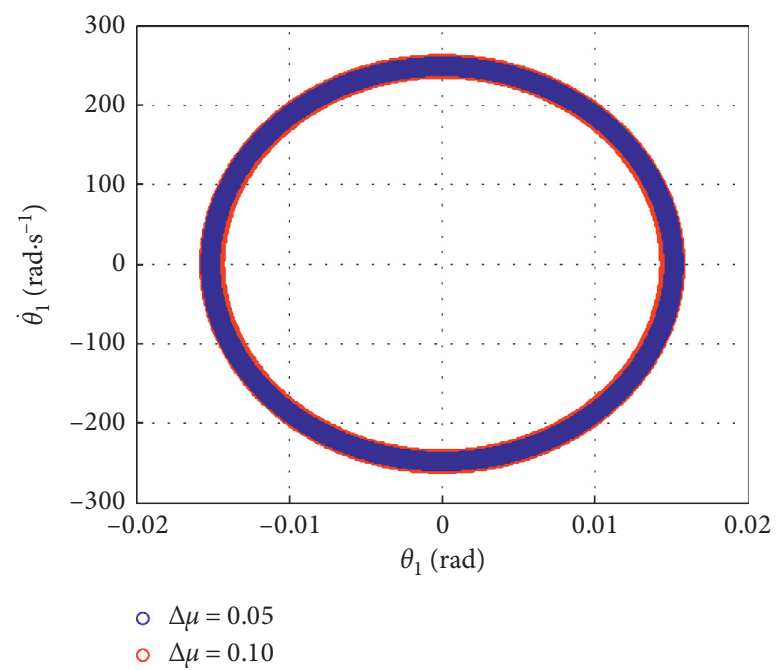

(c)

Figure 5: Dispersion of the limit cycles determined with $(\mathrm{CHBM}+\mathrm{ngPC})$ with $\mu m=0.25$ (a), $\mu m=0.35$ (b), and $\mu m=0.50$ (c) $(r=8$; $\left.\Delta V_{m, i}=0.01 ; \Delta \mu=0.05 ; 0.10\right)$.

\section{Conclusion}

This article has presented the analysis of the vibration behaviour of a clutch system with 6 DOF to take the uncertainties of parameters into account. Two approaches have been investigated. The first approach is to use ngPC directly on the displacements and velocities in order to determine the limit cycles. This method is only effective when there is one uncertain parameter (friction coefficient). For more complex systems with several DOF with a high number of uncertain parameters, this approach becomes inefficient, because the number of samples of direct simulations necessary to build stochastic modes would be too high. The second approach which combines the constrained harmonic balance method and nonintrusive generalized polynomial chaos is proposed as an alternative. The influence of the dimension of the interval dispersion of the uncertain parameters, the nominal value of the friction coefficient relative to the Hopf bifurcation point, and the number of uncertain parameters (from 1 to 8 ) has been studied.

The results demonstrate the ability of the approach which combines the $\mathrm{CHB}$ method with that of ngPC to properly estimate the dispersion of the limit cycles of a clutch system, especially for high values of the friction coefficient (far from the Hopf bifurcation point) when vibration levels are high even with relatively important uncertainties for the parameters. In this approach, the calculation costs of the limit cycles are significantly reduced.

\section{Data Availability}

The data used to support the findings of this study are included within the article.

\section{Conflicts of Interest}

The authors have no conflicts of interest to declare.

\section{References}

[1] B. Hervé, J.-J. Sinou, H. Mahé, and L. Jézéquel, "Extension of the destabilization paradox to limit cycle amplitudes for a nonlinear self-excited system subject to gyroscopic and circulatory actions," Journal of Sound and Vibration, vol. 323, no. 3-5, pp. 944-973, 2009.

[2] P. Wickramarachi and R. Singh, "Analysis of friction induced vibration leading to Eek Noise in a dry friction clutch," in Proceedings of the Internoise, Dearborn, MI, USA, 2002.

[3] G. Fritz, J.-J. Sinou, J.-M. Duffal, and L. Jézéquel, "Investigation of the relationship between damping and mode-coupling patterns in case of brake squeal," Journal of Sound and Vibration, vol. 307, no. 3-5, pp. 591-609, 2007.

[4] G. Fritz, J.-J. Sinou, J.-M. Duffal, and L. Jézéquel, "Effects of damping on brake squeal coalescence patterns-application on a finite element model," Mechanics Research Communications, vol. 34, no. 2, pp. 181-190, 2007.

[5] B. Hervé, J.-J. Sinou, H. Mahé, and L. Jézéquel, “Analysis of squeal noise in clutches and mode coupling instabilities including damping and gyroscopic effects," European Journal of Mechanics A/Solids, vol. 27, no. 2, pp. 141-160, 2008.

[6] E. Sarrouy, O. Dessombz, and J.-J. Sinou, "Stochastic study of a non-linear self-excited system with friction," European Journal of Mechanics-A/Solids, vol. 40, pp. 1-10, 2013.

[7] L. Nechak, S. Berger, and E. Aubry, "A polynomial chaos approach to the robust analysis of the dynamic behaviour of friction systems," European Journal of Mechanics-A/Solids, vol. 30, no. 4, pp. 594-607, 2011.

[8] L. Nechak, S. Berger, and E. Aubry, "Prediction of random self friction-induced vibrations in uncertain dry friction systems using a multi-element generalized polynomial chaos approach," ASME Journal of Vibration and Acoustics, vol. 134, 2012.

[9] N. Coudeyras, J.-J. Sinou, and S. Nacivet, "A new treatment for predicting the self-excited vibrations of nonlinear systems with frictional interfaces: the Constrained Harmonic Balance Method, with application to disc brake squeal," Journal of Sound and Vibration, vol. 319, no. 3-5, pp. 1175-1199, 2009. 
[10] M. Berveiller, B. Sudret, and M. Lemaire, "Stochastic finite elements: a non intrusive approach by regression," European Journal of Computational Mechanics, vol. 15, no. 1-3, pp. 81-92, 2006.

[11] G. Blatman and B. Sudret, "Sparse polynomial chaos expansions and adaptive stochastic finite elements using a regression approach," Comptes Rendus Mécanique, vol. 336, no. 6 , pp. $518-523,2008$.

[12] F. Chevillot, J.-J. Sinou, and N. Hardouin, "Nonlinear transient vibrations and coexistences of multi-instabilities induced by friction in an aircraft braking system," Journal of Sound and Vibration, vol. 328, no. 4-5, pp. 555-574, 2009.

[13] M. H. Trinh, S. Berger, and E. Aubry, "Non-intrusive generalized polynomial chaos takes uncertain parameters into account in the stability analysis of a clutch system," in Proceedings of the Congrès Français de Mécanique, pp. 1-6, Jeju, Korea, 2013.

[14] J.-J. Sinou, F. Thouverez, and L. Jézéquel, "Methods to reduce non-linear mechanical systems for instability computation," Archive of Computation Methods in Enginering, vol. 11, no. 3, pp. 257-344, 2004.

[15] B. Hervé, J.-J. Sinou, H. Mahé, and L. Jézéquel, "Extension of the destabilization paradox to limit cycle amplitudes for a nonlinear self-excited system subject to gyroscopic and circulatory actions," Journal of Sound and Vibration, vol. 323, no. 3-5, pp. 944-973, 2009.

[16] M. H. Trinh, S. Berger, and E. Aubry, "Stability analysis of a clutch system with multi-element polynomial chaos," Mechanics \& Industry, vol. 17, no. 2, p. 205, 2016. 